\title{
Magnetocaloric effect in temperature-sensitive magnetic fluids
}

\author{
KINNARI PAREKH, R V UPADHYAY and R V MEHTA* \\ Department of Physics, Bhavnagar University, Bhavnagar 364 002, India
}

MS received 27 August 1999; revised 25 January 2000

\begin{abstract}
The magnetocaloric properties of three different temperature-sensitive magnetic fluids were studied. The pyromagnetic coefficient for all the materials were obtained and it was found that this property depends on physical and magnetic properties like size, magnetization and Curie temperature. A theoretical model was developed to explain the behaviour of change in entropy with temperature.
\end{abstract}

Keywords. Magnetic fluid; pyromagnetic coefficient; Curie temperature.

\section{Introduction}

A magnetic fluid also known as a ferrofluid is a suspension of nanosize ferromagnetic or ferrimagnetic particles in a carrier liquid. Upon application of magnetic field, the entropy associated with the magnetic degree of freedom is changed due to the field-induced ordering. If performed adiabatically, this will result in a temperature change of a material, $\Delta T$, referred to as the magnetocaloric effect. The magnitude of this effect depends on the physical and magnetic properties like size, temperature dependence of magnetization, heat capacity of the material and carrier liquid. In this paper, we report the magnetocaloric properties of certain temperature-sensitive magnetic fluids. Here, an indirect measurement technique to determine the change in entropy using temperature-dependent magnetic property of magnetic fluid, is reported.

\section{Theoretical analysis}

When a material is magnetized by application of a magnetic field, the entropy associated with the magnetic degree of freedom, $S_{\mathrm{m}}$, is changed, as the field changes the magnetic order of the material. Under adiabatic conditions, $\Delta S_{\mathrm{m}}$ must be compensated for by an equal but opposite change in the entropy associated with the lattice, $\Delta S_{1}$, resulting in a change in temperature of the material, $\Delta T$. This effect is referred to as the magnetocaloric effect. The magnetocaloric effect can be related to the magnetic properties of the material through the thermodynamic Maxwell's equation

$$
\left(\frac{\partial S}{\partial H}\right)_{T}=\left(\frac{\partial M}{\partial T}\right)_{H} .
$$

\footnotetext{
*Author for correspondence
}

From the magnetic properties, the isothermal entropy change of a material can be calculated using this relation as

$$
\Delta S_{\mathrm{m}}=\int_{0}^{H}\left(\frac{\partial M}{\partial T}\right)_{H} \mathrm{~d} H .
$$

The magnetization measurement made at discrete temperature intervals, $\Delta S_{\mathrm{m}}$, can be approximated as

$$
\begin{aligned}
\Delta S_{\mathrm{m}}= & \frac{1}{\Delta T}\left[\int_{0}^{H} M(T+\Delta T, H) \mathrm{d} H\right. \\
& \left.-\int_{0}^{H} M(T, H) \mathrm{d} H\right] .
\end{aligned}
$$

The above equation shows that the change in entropy of a system is equivalent to the area between the two magnetic isotherms divided by the temperature difference between the isotherms. This formulation is useful for graphical or numerical calculations of $\Delta S_{\mathrm{m}}$ from the magnetization data (McMichael et al 1993). In the case of a paramagnet, the temperature and field dependence of magnetization, $M$, is described by the Langevin's function

$$
M=N \mu[\operatorname{coth} \alpha-1 / \alpha], \alpha=\mu(T) H / k T,
$$

where, $N$ and $\mu(T)$ are respectively the number and size of non-interacting magnetic moments in the system, $k$ the Boltzmann constant and $T$ the absolute temperature. If $\mu(T)$ is temperature independent, the magnetization measured at various temperatures and fields should fall on a single line when plotted as a function of $H / T$. Such a plot is a common test for the superparamagnetic behaviour. In a temperature-sensitive magnetic fluid, the magnetic moment varies with temperature and, hence, the magnetization data of the fluid cannot be expected to fall on a single line. Therefore, by substituting (4) in (2) and performing the integration, the isothermal entropy change of 
an ensemble of superparamagnetic particles is given by (Shull et al 1992)

$$
\Delta S=N k\left[1-\alpha \operatorname{coth} \alpha+\ln \left(\frac{\sinh \alpha}{\alpha}\right)\right] .
$$

Equation (5) is valid only for the monodispersed system. In a magnetic fluid, since all the nanomagnets are in a random position, and, if we neglect the intergrain interactions, it resembles a paramagnetic gas. Also since these grains possess giant magnetic moment, they are termed as superparamagnetic. Furthermore, because the magnetic moment of the particle is a function of working range of temperature, therefore, a significant change in entropy will be observed in a magnetic fluid. Thus in a magnetic fluid, the distribution of particle size generally follows the log-normal distribution function given as

$$
P(D)=\frac{1}{D \sigma(2 \pi)^{1 / 2}} \exp \left[-\frac{\left(\ln \left(D / D_{0}\right)\right)^{2}}{2 \sigma^{2}}\right],
$$

where, $\sigma$ is the standard deviation, $D$ the particle diameter, and $\ln D_{0}$ a mean of $\ln D$. Therefore, to determine the change in entropy in a fluid, (5) is corrected for the distribution function given by (6).

The entropy change is determined from the measurement of variation of fluid height as a function of temperature using Quincke's set-up.

\section{Experimental}

\subsection{Preparation of fine particles and magnetic fluid}

Three different magnetic fluids whose saturation magnetization significantly changes with temperature were syn-

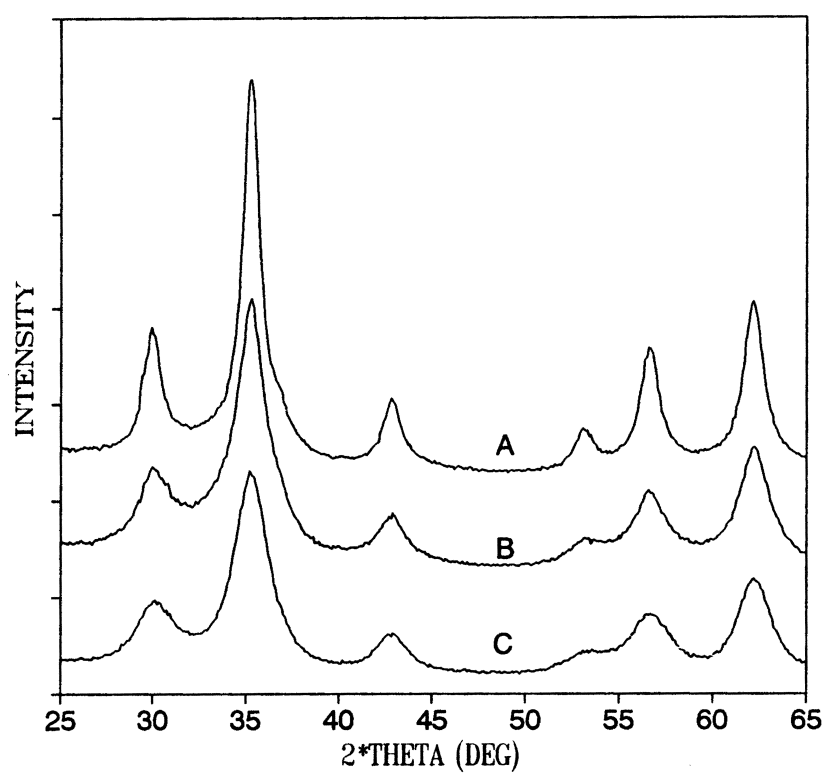

Figure 1. X-ray diffraction pattern for samples A, B and C. thesized using chemical co-precipitation technique. The fine particles of mixed ferrite samples of $\mathrm{Mn}-\mathrm{Zn}$ and $\mathrm{Fe}-\mathrm{Zn}$ possess a single phase spinel structure (figure 1). These particles were dispersed in a non-magnetic carrier liquid-diester. The technique of synthesization of such a fluid is given in figure 2 . The resulting fluids were stable and liquid at room temperature. The characteristic properties of all the fluids are given in table 1 .

\subsection{Magnetization measurement}

An Oxford instruments CF 1200 cryostat and Vibrating Sample Magnetometer (VSM) were used to carry out magnetization measurement at low temperature. The magnetometer was calibrated with the nickle standard.

\subsection{Quincke's set-up}

A double-walled U-shaped glass tube was used to measure the change in height of a fluid. To maintain a uniform temperature, a constant temperature bath having an accuracy of $\pm 1^{\circ} \mathrm{C}$ was used. While one limb of the U-tube was kept inside a magnetic field of $1 \mathrm{kOe}$, the other limb was at zero field. The details about this setup have been discussed in our previous paper (Parekh Kinnari and Upadhyay 1997).

\section{Results and discussion}

\subsection{Magnetization measurement}

Figure 3 shows the typical temperature-dependent magnetization curve. For all the samples, up to $80 \mathrm{~K}$ the system shows zero remanance, i.e. particles are superparamagnetic at the measuring time scale of $100 \mathrm{sec}$. To determine the various parameters like domain magnetization,

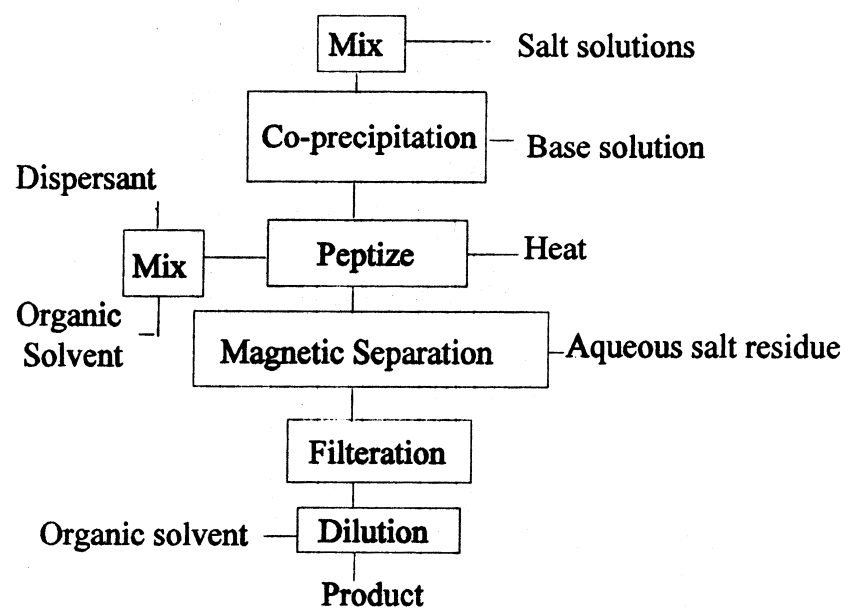

Figure 2. Flow chart for the preparation of a magnetic fluid. 
Table 1. Characteristic properties of fluids.

\begin{tabular}{lccccc}
\hline Sample & Code & $\begin{array}{c}D_{\mathrm{x}} \\
(\AA)\end{array}$ & $\begin{array}{c}\text { Carrier density } \\
(\mathrm{g} / \mathrm{cc})\end{array}$ & $\begin{array}{c}\text { Volume } \\
\text { fraction }\end{array}$ & $\begin{array}{c}\text { Viscosity } \\
\text { @ 37C (cP) }\end{array}$ \\
\hline $\mathrm{Mn}_{0.5} \mathrm{Zn}_{0.5} \mathrm{Fe}_{2} \mathrm{O}_{4}$ & $\mathrm{~A}$ & 67 & 0.94 & 0.0891 & 10.53 \\
$\mathrm{Fe}_{0.5} \mathrm{Zn}_{0.5} \mathrm{Fe}_{2} \mathrm{O}_{4}$ & $\mathrm{~B}$ & 50 & 0.94 & 0.0494 & 2.94 \\
$\mathrm{Fe}_{0.3} \mathrm{Zn}_{0.7} \mathrm{Fe}_{2} \mathrm{O}_{4}$ & $\mathrm{C}$ & 40 & 0.94 & 0.0699 & 4.97 \\
\hline
\end{tabular}
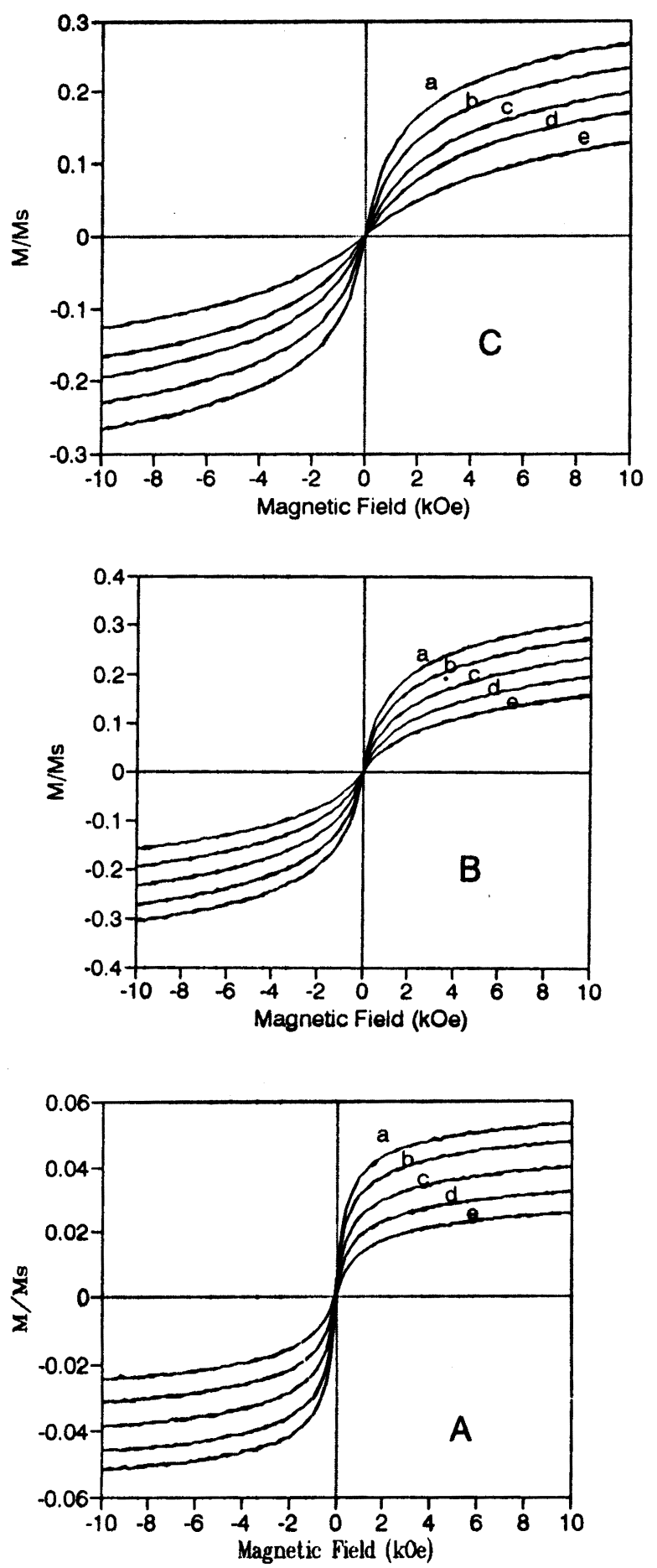

Figure 3. Typical temperature dependence magnetization curves for samples A, B, C at (a) $80 \mathrm{~K}$, (b) $120 \mathrm{~K}$, (c) $160 \mathrm{~K}$, (d) $200 \mathrm{~K}$ and (e) $240 \mathrm{~K}$.
$M_{\mathrm{d}}$, median particle size, $D_{\mathrm{m}}$, saturation magnetization of fluid, $M_{\mathrm{s}}$, etc the Langevin's function was used. Taking into account the polydispersity, the magnetization can be obtained as

$$
M=\int_{0}^{\infty} M_{\mathrm{s}} L(\alpha) P(D) \mathrm{d}(D) .
$$

The experimental magnetization data at $80 \mathrm{~K}$ were fitted to (7). The appropriate fit parameters obtained are given in table 2. The increase in temperature does not change the value of $D_{\mathrm{m}}$ (median diameter) and $\sigma$; only $M_{\mathrm{d}}$ value changes with the temperature. Therefore, the value of $M_{\mathrm{d}}$ was derived at each temperature and its variation with temperature are depicted in figure 4 . The fitting of these data shows that all the samples follow $T^{3 / 2}$ variation in the range $80-300 \mathrm{~K}$. The value of transition temperature $T_{\mathrm{C}}$ was obtained by extrapolating this line (table 2) (Assuming that the variation of magnetization follows the same trends).

\subsection{Quincke's measurement}

In this technique, the variation of magnetization with temperature can be directly correlated with the change in fluid height with temperature. The factors which are responsible for the change in fluid height are: (i) thermal expansion of the fluid, (ii) Brownian relaxation effect and (iii) variation of magnetic moment with temperature, i.e. the Langevin's function. The effect of the first term was corrected by considering the volumetric expansion of a fluid with temperature. In the present case, the effect of the second term has to be considered since only $1 \mathrm{kOe}$ field was applied. In this technique, the change in area under the magnetization curve at different temperatures, $\Delta \bar{M}\left[\bar{M}\left(T_{1}\right)-\bar{M}\left(T_{2}\right)\right]$, were obtained from the relation (Parekh Kinnari and Upadhyay 1997)

$$
\Delta \bar{M}=\rho_{1} g / \mu_{0} H\left[k_{1}-k_{2}\left\{1+\rho_{2} / \rho_{1}\right\}\right]
$$

where, $k_{1}, k_{2}$ and $\rho_{1}, \rho_{2}$ are corresponding height and density of the fluid at temperature $T_{1}$ and $T_{2}, g$ is the gravitational acceleration, $\mu_{0}$ the permeability of free space and $\bar{M}$, the field averaged magnetization given as

$$
\bar{M}=\frac{1}{H} \int_{0}^{H} M \mathrm{~d} H .
$$


Table 2. Results from magnetization and Quincke's measurement.

\begin{tabular}{lcccccc}
\hline Code & $\begin{array}{c}M_{\mathrm{d}} \\
(\mathrm{e} m \mathrm{c} / \mathrm{cc})\end{array}$ & $\begin{array}{c}D_{\mathrm{m}} \\
(\AA)\end{array}$ & $\sigma$ & $\begin{array}{c}T_{\mathrm{C}}(K) \\
(\mathrm{Mag})\end{array}$ & $\begin{array}{c}T_{\mathrm{C}}(K) \\
(\text { Quincke })\end{array}$ & $\begin{array}{c}\text { p.c. } \\
(\mathrm{emu} / \mathrm{cc} K)\end{array}$ \\
\hline A & 210 & 71 & $0 \cdot 28$ & 340 & 360 & $5 \cdot 125$ \\
B & 200 & 57 & $0 \cdot 37$ & 364 & 460 & $3 \cdot 125$ \\
$\mathrm{C}$ & 100 & 43 & $0 \cdot 25$ & 347 & 370 & $2 \cdot 128$ \\
\hline
\end{tabular}
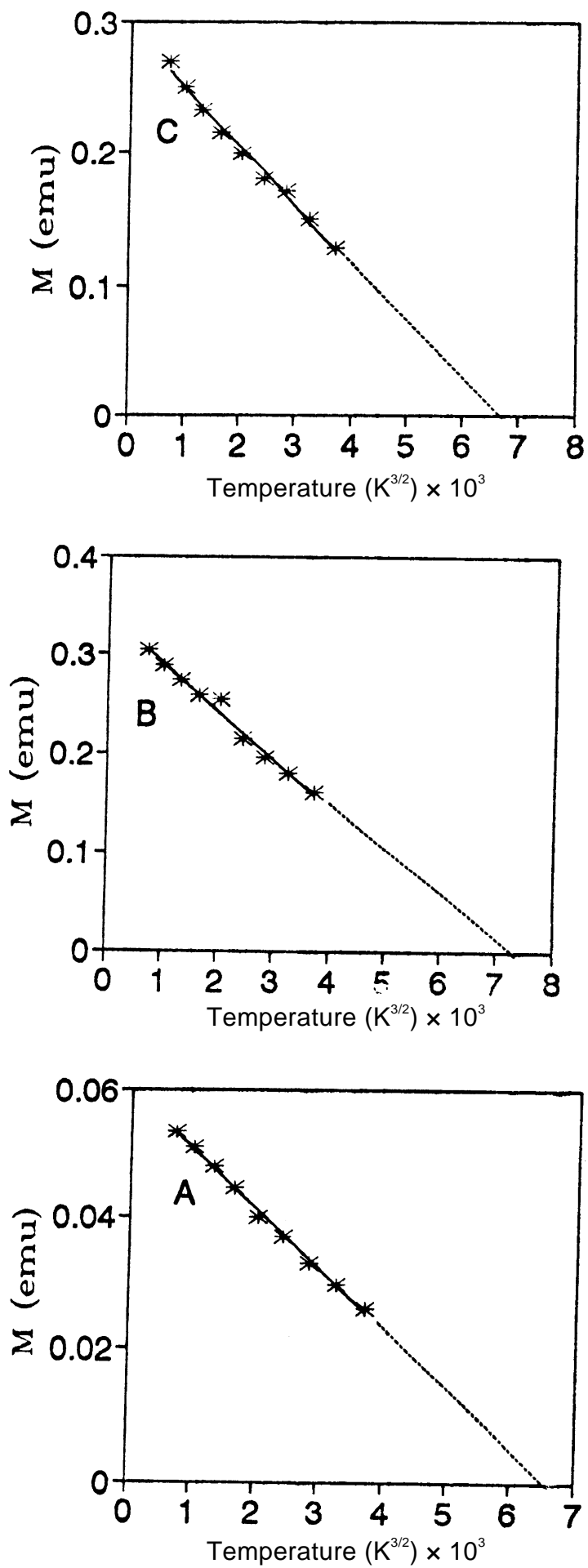

Figure 4. $T^{3 / 2}$ dependence curves for all the samples.

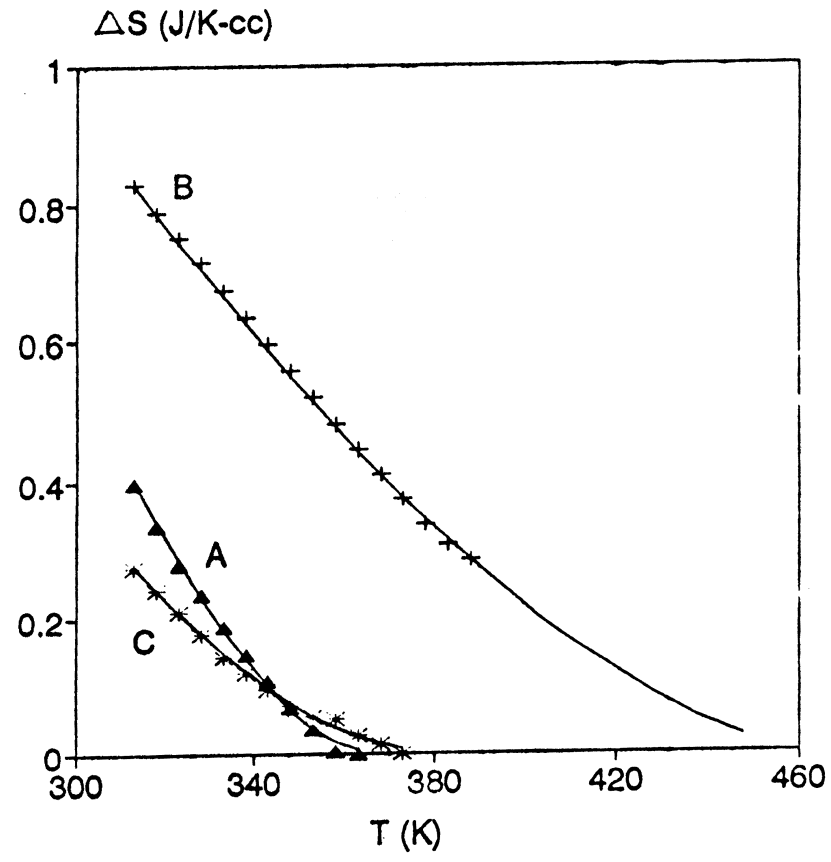

Figure 5. Change in entropy versus temperature curves. The solid line is fit to (5) and (6).

The experimental results obtained from (8) were fitted with an empirical relation as

$$
\Delta \bar{M}=K\left[x-\left[\frac{T-T_{0}}{T_{0}}\right]^{3 / 2}\right] .
$$

Here, $K$ and $x$ are constants and depend upon the characteristics of the fluid and $T_{0}$ the ordering temperature. The value of Curie temperature obtained from this fitting is the point of intersection of the initial straight line and the tangent drawn from the saturating points. The values of $T_{\mathrm{C}}$ thus obtained are in good agreement with those obtained from the other measurements like SANS (Upadhyay Trupti et al 1997), ESR (Parekh Kinnari et al 1999) etc. Though this technique will give reasonably accurate value of transition temperature, the mere fitting of this empirical relation does not ascertain any physical importance regarding the fit parameters $K, x$ and $T_{0}$. In addition to this, the variation of magnetization in the region near to the Curie temperature is a function of sample characteristics. At low temperature, the sample may obey the $T^{3 / 2}$ dependence, but for high temperature the drop of magnetization becomes fast which can follow the $T^{n}$ law, where $n$ can 
have any value. Since the value of pyromagnetic coefficient, $(\partial M / \partial T)_{H}$, (p.c.) is obtained from the slope of the curve of $M_{\mathrm{d}}$ vs $T$, the precise value of p.c. cannot be determined using this technique, which is an important parameter for a temperature-sensitive magnetic fluid. In order to derive these parameters from the data, we have used the data of change in fluid height with temperature, as this is equal to the area under the magnetization curve, and (5) and (6).

Figure 5 shows the variation of entropy change versus temperature for all three samples. The line is a theoretical fit from which the value of $M_{\mathrm{d}}, D_{\mathrm{m}}$, and $\sigma$ were derived, which agrees with those obtained from the magnetization data. In each case, the variation of $M_{\mathrm{d}}$ with temperature was obtained and it was observed that for $T>300 \mathrm{~K}$, it varied linearly with $T$. The value of p.c. for each fluid was calculated using the $M_{\mathrm{d}}$ vs $T$ curve (table 2). The results of these measurements show that sample A has higher value of p.c. compared to the other samples.

\section{Conclusion}

It is possible to study the entropy change in a magnetic fluid using Quincke's measurements. This makes this technique a complementary tool to obtain the material- related properties like domain magnetization, particle size, size distribution, Curie temperature and the pyromagnetic coefficient of the fluid.

\section{Acknowledgements}

One of the authors (RVU) is thankful to Dr S W Charles, UCNW, Bangor, UK, for providing the low temperature VSM facilities. This work is supported under IUC-DAEF project No. CRS-M 36.

\section{References}

McMichael R D, Ritter J J and Shull R D 1993 J. Appl. Phys. 736946

Parekh Kinnari and Upadhyay R V 1997 Indian J. Pure Appl. Phys. 35523

Parekh Kinnari, Upadhyay R V, Mehta R V and Srinivas D 1999 J. Appl. Phys. (communicated)

Shull R D, McMichael R D, Swartzendruber L J and Bennett L H 1992 Studies of magnetic properties of fine particles and their relevance to materials science (eds) J L Dormann and D Fiorani (Elsevier Science Publishers B V) p.161

Upadhyay Trupti, Upadhyay R V and Mehta R V 1997 Phys. Rev. B55 5585 